\title{
Local anodic oxidation of graphene layers on $\mathrm{SiC}$
}

\author{
B.R. Borodin, M.S. Dunaevskiy, P.A. Alekseev
}

Ioffe Institute, 194021, St. Petersburg, Russia

e-mail: brborodin@gmail.com

Graphene is a promising material for the creation of electronic and optoelectronic devices of a new generation. To be cost-effective, large-sized layers of high-quality homogeneous graphene are required. High-quality epitaxial graphene can be grown by thermal destruction of a semiinsulating monocrystalline silicon carbide substrate ( $\mathrm{SiC}$ ) [1]. Most lithographic techniques involve the application and removal of resist from the surface of graphene, that reduce the mobility of charge carriers [2]. An alternative method of lithography, excluding the use of resist, is the local anodic oxidation (LAO) [3]. In this method, an electrical voltage between the probe of the atomic force microscope (AFM) and the graphene with water film on the surface leads to the local oxidation of the graphene.

The aim of our work was to perform the LAO of a graphene layers on SiC and to study electronic properties of the graphene and graphene oxide by Kelvin probe microscopy (KPM) and conductive AFM. The experiment was performed with a Ntegra Aura (NT-MDT) microscope using DCP (NT-MDT) and HA_FM / W2C (NT-MDT) conducting probes with a tip diameter of $100 \mathrm{~nm}$ and $20 \mathrm{~nm}$, respectively. The sample was a monolayer graphene with a small fraction $(\sim 10 \%)$ of two-layered islands with submicron dimensions, obtained by thermal destruction in argon of the Si-face of the $6 \mathrm{H}-\mathrm{SiC}$ substrate (0001) [1]. By the method of local anodic oxidation, graphene oxide regions were obtained. The KPM study showed that oxided region have a $350 \mathrm{mV}$ higher surface potential than the graphene. The I-V curves measured by the C-AFM revealed the semiconducting character of the graphene oxide. In addition, it was studied the LAO regimes for the creation of the graphene nanoribbon and nanoconstriction with the minimal width. The detection of oxidized regions was performed by measuring the frictional force of the probe on the surface. In Figure 1 the maps of the distribution of the frictional force of a surface are presented. Light areas on the image correspond to oxidized graphene, dark areas to not oxidized. Figures show a graphene nanoribbon with a width of $20 \mathrm{~nm}$ (Fig. 1a) and a $10 \mathrm{~nm}$ wide nanoconstriction (Fig. 1b), which is comparable with the best results obtained on peeled graphene.
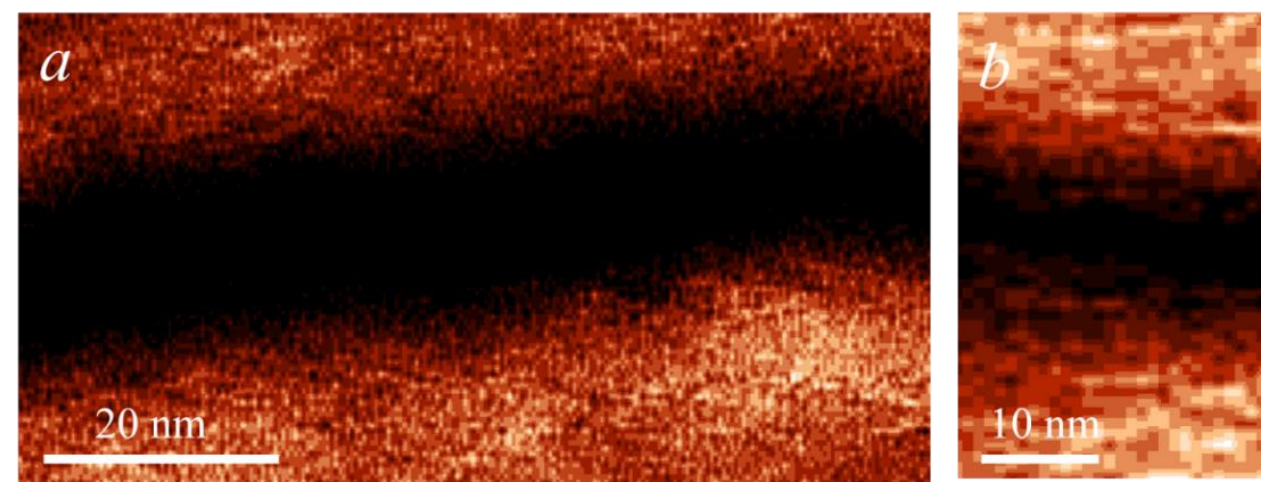

Figure 1. (a) Graphene nanoribbon and (b) graphene nanoconstriction (width 10nm).

To conclude, electrical properties of the graphene oxide layers formed by the LAO were studied. The results of this work allow us to talk about the LAO of graphene on SiC, as a method of nanolithography with a resolution of $10 \mathrm{~nm}$, which can be used to create devices based on thin layers of graphene.

This work is supported by Presidential Grant MK-5852.2018.2.

1. V.Y. Davydov, D.Y. Usachov, S.P. Lebedev, et al., Semiconductors 51, 1072 (2017).

2. H. Song, S. Li, H. Miyazaki, et al., Sci. Rep. 2, 337 (2012).

3. F. Colangelo, V. Piazza, C. Coletti, et al., Nanotechnology 28, 105709 (2017). 